\title{
TIPIFICACIÓN DE LA GALLINA CRIOLLA EN LOS AGROECOSISTEMAS CAMPESINOS DE PRODUCCIÓN EN LA ZONA DE INFLUENCIA DE LA SELVA DE FLORENCIA (CALDAS)
}

\author{
Jorge Luis Tovar-Paredes ${ }^{1}$, William Narváez-Solarte ${ }^{2}$, \\ Liceth Agudelo-Giraldo ${ }^{3}$
}

Recibido el 14 de marzo de 2014, aprobado el 14 de agosto de 2014 y actualizado el 27 de abril de 2015

DOI: 10.17151/luaz.2015.41.4

\section{RESUMEN}

La avicultura de traspatio es una actividad pecuaria de gran importancia en las comunidades rurales del país, caracterizada por la baja inversión requerida y por la facilidad para efectuarla; las especies más utilizadas son las criollas dado que se adaptan a las condiciones adversas para su crianza, esta actividad fortalece el bienestar de las familias campesinas que aprovechan al máximo la mano de obra familiar y es una fuente generadora de bienes para el hogar campesino. Objetivo: Caracterizar los diferentes sistemas de producción avícola de traspatio en la zona de influencia de la Selva de Florencia del municipio de Samaná del departamento de Caldas. Metodología: De las 103 fincas del área de estudio se encuestaron 76 fincas, correspondientes a la totalidad de los predios con producción de gallinas criollas, en las que se recolectó la información en un formato integrado por variables cuantitativas y cualitativas, de los aspectos socioculturales, económicos, medioambientales, y de manejo de las aves, que permitieron determinar la interacción de estas y así agrupar los diferentes sistemas de producción. El análisis de la información se realizó mediante el análisis de clusters a partir de la aplicación de técnicas multivariadas. Se consideraron las 25 variables cuantitativas de mayor poder discriminatorio y se redujo la dimensionalidad del problema, descartando aquellas variables de bajo poder discriminante. Resultados: El análisis permitió identificar nueve componentes principales, que explican el $71 \%$ de la varianza total; para así establecer seis sistemas finca, donde las aves representan desde el $65 \%$ hasta el 2,8\% del capital pecuario, en relación directa con la extensión de la finca y la vocación agrícola principal. Conclusiones: La explotación de gallinas es una actividad de importancia en las comunidades rurales, que se complementa con otras actividades productivas realizadas en el campo. Aporta nutrientes a la dieta cumpliendo la función de transformar productos de origen vegetal y residuos en proteína de origen animal.

\section{PALABRAS CLAVE}

Aves, caracterización, avicultura, traspatio, autóctonas. 


\title{
CHARACTERIZATION OF THE CREOLE HEN IN THE PEASANT PRODUCTION AGRO- ECOSYSTEM IN THE RAIN- FOREST AREA OF INFLUENCE IN FLORENCIA (CALDAS)
}

\begin{abstract}
Backyard hen farming is an important farming activity in the rural communities in the country characterized by the low investment required and for the easiness to carry it out. Creole hens are mostly used because of their adaptability to adverse conditions for breeding. This activity strengthens the peasant families' welfare since they take the most out of the family workforce and it is a generating source for peasant household goods. Objective: To characterize the different backyard hen production systems in the rainforest influence area of Florencia, municipality of Samaná in the Department of Caldas. Methodology: From the 103 farms in the area of study, 76 farms corresponding to all the properties with Creole hen farming were surveyed and information was collected using a quantitative and qualitative integrated variables format dealing with socio-cultural, economic, environmental and chicken management aspects which allowed determining their interaction and grouping the different production systems. The analysis of the information was carried out through cluster analysis parting from multivariate techniques application. The Twenty-five qualitative variables with the highest discriminatory power were considered and the dimensionality of the problem was reduced discarding those variables with a low discriminant power. Results: The analysis allowed identifying nine main components that explain $71 \%$ of the total variance in order to establish six farm systems where the hens represent from $65 \%$ to $2.8 \%$ of the farming capital in direct relation with the farm extension and the main farming vocation. Conclusions: Hen production is an important activity in rural communities which is complemented with other productive activities developed in the land. It contributes with nutrients to the diet accomplishing the function of transformation of vegetal origin products and residues into animal origin protein.
\end{abstract}

\section{KEY WORDS}

Birds, characterization, chicken, backyard, native.

\section{INTRODUCCIÓN}

Más del $80 \%$ de las familias rurales de los países en desarrollo, mantienen gallinas en las huertas de sus casas, entre las que se destacan las estirpes criollas, conservadas por los campesinos, tanto por la necesidad de generar ingresos, obtener productos proteicos de alto valor biológico o por simple tradición cultural (Pym, 2013).

Los factores socioculturales, económicos y técnicos han determinado la conformación de los actuales sistemas de 
producción con secuencia histórica, con un mayor desarrollo en unos sitios que en otros, moviéndose entre la agricultura extensiva y la intensiva, conjugando actividades interdependientes para la utilización de la tierra, que solo pueden ser entendidas si se considera a la parte como un todo del sistema y del modelo de organización social (Rodríguez y Carvajal, 1998).

La Selva de Florencia, se encuentra ubicada en la Cordillera Central al Oriente del departamento de Caldas, considerada como el último fragmento de Bosque Pluvial Andino del cinturón cafetero en dicha formación montañosa. Está conformada en gran parte de Selva Andina y sus alrededores están compuestos por potreros y cultivos de pancoger. Además de la abundancia de agua e importancia para la generación de energía eléctrica de la región y el país, esta es uno de los pocos reductos de biodiversidad que posee el departamento (Rivera y Rivera, 1995; Corpocaldas y Universidad de Caldas, 2001; Ballesteros et al., 2006). Esta región presenta variedad de sistemas de producción como respuesta a la heterogeneidad de complejas relaciones socioeconómicas, que articulan zonas geográficas en presencia de conflictos sociales donde el Estado no hace presencia con políticas definidas y programas de desarrollo acordes a la problemática de la región.

Colombia cuenta con una población avícola de traspatio del género Gallus distribuida principalmente en la región Andina, criadas en libertad en los huertos, con alimentos del medio como: lombrices, insectos, etc. Los huevos producidos se destinan para el consumo del núcleo familiar y parte para el repoblamiento del píe de cría (Valencia y Betancourth, 1991; Álvarez, 1998). La producción de carne y huevo procedente de este tipo de explotación es afectada por la ausencia de normas técnicas y de sanidad adecuadas, que la convierten en un tipo de producción de alto riesgo sanitario para la avicultura industrial. Sin embargo, la FAO, dentro el programa especial para la seguridad alimentaria, muestra gran interés en desarrollar la producción de aves de corral a nivel familiar, como herramienta para el aprovechamiento y transformación de los subproductos de la finca y como estrategia para mejorar los índices de desnutrición de la población, para el desarrollo de una agricultura sostenible (Farrel, 2014).

El objetivo de la presente investigación es caracterizar la participación ecológica de la gallina criolla en los sistemas de producción campesinos que incluye la forma de explotación, el nivel tecnológico, los recursos utilizados y el aspecto sociocultural.

\section{MATERIALES Y MÉTODOS}

El trabajo se realizó en la zona de influencia de la Selva de Florencia del municipio de Samaná, departamento de Caldas de la República de Colombia, ubicada a 74050' de longitud Oeste y 
5032' de latitud Norte, entre los 700 y 2.300 msnm, con precipitación anual de $6.270 \mathrm{~mm}$, temperatura media de $20^{\circ} \mathrm{C}$ y una superficie aproximada de $240 \mathrm{~km}^{2}$.

Se encuestaron 73 granjas, número correspondiente a la totalidad de las fincas con producción de gallina criolla, de una población de 103 granjas que conforman el área de estudio, en las que se recolectó la información en un formato integrado por variables cuantitativas y cualitativas, de los aspectos socioculturales, económicos, medioambientales, y de manejo de las aves, que permitieron determinar la interacción entre ellas y así agrupar los diferentes sistemas de producción. El análisis de la información se realizó mediante el agrupamiento de las fincas con mínima variabilidad ínter-clases, mediante el análisis de clusters a partir de la aplicación de técnicas multivariadas, utilizando el programa estadístico SAS (2008).

Para el análisis se consideraron las 25 variables cuantitativas de mayor poder discriminatorio que fueron: área de la finca, distancia al pueblo, tiempo que vive en la finca, número de personas, área en caña, área en plátano, área en café, área en maíz, área en yuca, número de cerdos, número de bovinos, número de equinos, peso promedio de aves machos, peso promedio de aves hembras, producción de huevos, incubación, venta de huevos, compra de huevos criollos, compra de huevos comerciales, procedencia de gallinas, costo de alimento por ave, costo de alimento por huevo, número de gallos, número de gallinas y número de gallinas que criaría; y se redujo la dimensionalidad del problema, descartando aquellas variables de bajo poder discriminante, utilizando como primer criterio de descarte el coeficiente de variación inferior al $60 \%$ y como segundo el coeficiente de correlación superior al 50\%, en cuyo caso se descartó la variable dependiente.

\section{RESULTADOS}

\section{Análisis de los grupos o sistemas de producción}

El análisis permitió identificar nueve componentes principales, que explican el $71 \%$ de la varianza total; y así conformar seis conglomerados o sistemas de producción.

\section{Grupo 1. Sistema finca de pequeñas extensiones con cobertura en café y capital pecuario pequeño representado en un $65 \%$ en aves}

Este grupo reúne el $40,4 \%$ de las fincas estudiadas, los predios tienen un área promedio de 2,5 ha. El capital pecuario de este grupo asciende aproximadamente a USD\$ 42,45, correspondientes al $7,7 \%$ de los ingresos brutos totales de la producción agropecuaria de la finca, el mayor aporte lo hacen las gallinas con el $65 \%$, los cerdos con el $20,66 \%$ y los bovinos con el $14,34 \%$. Se manejan parvadas de 5 a 6 gallinas. La 
alimentación de las aves es diversificada de acuerdo a la edad. Durante el crecimiento las pollitas consumen maíz molido, residuos de cocina y alimento comercial (Tabla 1); al crecer la alimentación de las gallinas depende de la recolección de insectos, gusanos, lombrices, hojas tiernas, estiércol, desperdicios y maíz en grano que se suministra como complemento a la ración. En la fase de producción, las aves reciben la misma dieta de la fase de crecimiento, complementada con alimento comercial (Tabla 2).

Las aves presentan características fenotípicas de gallinas "semipesadas"; con diferentes colores, un peso promedio a la edad adulta de $1,8 \mathrm{~kg}$ las hembras y $2,0 \mathrm{~kg}$ los machos. El $77 \%$ de estas aves provienen de la incubación natural en la misma finca y el $23 \%$ del intercambio con los vecinos. Las aves alcanzan la madurez sexual a los 7 meses de edad, con una producción anual de 175 huevos, de los cuales el $80 \%$ se destina para autoconsumo y el $20 \%$ para la incubación; el peso medio del huevo es de $57 \mathrm{~g}$ y la eclosión del 65\%. La selección genética de los animales se realiza por tamaño del animal como único parámetro de selección.

El índice de mortalidad de las aves es de 25\%. Entre las principales causas del deceso se encuentran las afecciones respiratorias, diarreas, viruela aviar, parásitos internos y externos. Para el tratamiento de las enfermedades se utilizan antibióticos como la oxitetraciclina y ambramicina; el metronidazol contra parásitos internos; el limón y el cañaguate (Tecoma spectabilis) para disminuir la fiebre. Es de destacar que no tienen establecido planes de vacunación, para estos animales, ni tienen conocimiento de los conceptos básicos de la bioseguridad, dentro de la producción animal.

El costo de producción por huevo es de USD $\$ 0,020$ representados principalmente en maíz y alimento balanceado comercial. El costo de la mano de obra representa alrededor de USD\$ 0,96 por gallina al mes, correspondiente a los 20 o 30 minutos gastados en el manejo de esta especie diariamente. Para suplir las necesidades alimenticias, la familia consume una gallina por mes y compra aproximadamente 24 huevos en el mismo periodo, de los cuales el $70 \%$ son de gallinas criollas que se adquieren en la misma zona a un precio de USD\$ 0,074, que les permite alcanzar un consumo anual de 129 huevos per cápita.

Las mujeres encargadas del bienestar de las aves, tienen en promedio 47 años de edad, las protegen de los depredadores y de las inclemencias del medio ambiente con jaulas construidas en guadua y plástico; los nidos son rústicos, localizados dentro de la vivienda para evitar la depredación de los huevos por perros y animales silvestres. Los recipientes donde se ofrece la comida son de diferentes materiales, el agua de bebida es obtenida por las aves directamente de las acequias, riachuelos o charcos. El deseo de estas mujeres es el de tener una parvada de 22 gallinas criollas o de líneas comerciales para evitar la 
compra de huevos y contribuir a la seguridad alimentaria del núcleo familiar.

Grupo 2. Sistema finca de pequeñas extensiones dedicado a la producción de café y caña de azúcar, con capital pecuario pequeño del cual el $42 \%$ está representado en aves

Este grupo reúne el $10,6 \%$ de las fincas, los predios tienen un área promedio de 2,6 ha. La actividad pecuaria genera aproximadamente USD $\$ 70,49$ de ingresos brutos al año, correspondientes al $10,37 \%$ de los ingresos de la actividad agropecuaria, de los cuales el $42 \%$ está representado en aves, el $36,5 \%$ en bovinos y el $21,5 \%$ en cerdos.

La gallina, especie más representativa, se maneja en parvadas de 8 animales sin gallo en forma tradicional, fenotípicamente poseen características de semipesadas, tiene un peso a la edad adulta de $2,0 \mathrm{~kg}$. El $68 \%$ de estas aves provienen de la genética de la misma finca mediante incubación natural la que alcanza el $45 \%$ de eclosión, la selección se hace por tamaño y color del plumaje de los animales, el $32 \%$ restante es producto del intercambio con los vecinos, este tipo de aves alcanza la madurez sexual a los 7 meses de edad. El índice de producción de huevos alcanza el 28\%, que representa 47 huevos al mes, con peso promedio de $60 \mathrm{~g}$. El 74\% de la producción se destina para autoconsumo y el $26 \%$ para incubación. Las aves se alimentan uno o dos veces por día, básicamente con maíz y se les administra alimento balanceado comercial en pequeñas cantidades. La mortalidad alcanza el $18 \%$ en fase de cría, causada por afecciones respiratorias, diarreas y viruela aviar. La oxitetraciclina y la penicilina son los fármacos preferidos; el limón es utilizado como un brebaje, no se practica la vacunación. El costo de producción de un huevo es de USD\$ 0,032 representados en maíz. La mano de obra mensual utilizada en el cuidado de los animales se estima USD\$ 0,087 por gallina al mes.

Las familias complementan las necesidades de alimentos con la compra de 14 huevos comerciales por mes, adquiridos en la tienda del casco urbano a un precio de USD\$ 0,074. Lo que les permite lograr un consumo per cápita de 101 huevos por año.

Las aves de este grupo están a cargo de mujeres que tienen en promedio 44 años de edad, manejan las aves en un sistema extensivo. Una de las prioridades de la familia de este grupo es llegar a tener una parvada de 25 gallinas criollas manejadas con algunos criterios técnicos, para generar ingresos y evitar la compra de huevos.

Grupo 3. Sistema de fincas integrales pequeñas, donde la producción avícola representa el $25,3 \%$ del capital pecuario

Este grupo reúne el 8,5\% de los predios, el área promedio de las fincas está alrededor de 0,75 ha. La actividad agrícola aporta aproximadamente USD\$233 de ingresos brutos por año. Las 
aves representan el $65,8 \%$ del capital pecuario productivo, con USD\$ 26,83; mientras los cerdos representan el 10,76\%.

Manejan parvadas de 11 gallinas, las cuales presentan características fenotípicas de aves semipesadas y de diferentes variedades, el peso de los animales a la edad adulta es de $2,0 \mathrm{~kg}$ tanto para hembras como para machos; el $79 \%$ de los animales proviene del mercado local, el $12,5 \%$ de la incubación natural en la misma finca y el $8,5 \%$ del intercambio con los vecinos. Las gallinas alcanzan la madurez sexual a los seis meses de edad y logran una producción de 50,1\% que representa 116 huevos al mes, de $56 \mathrm{~g}$ de peso cada uno, de los cuales el $60 \%$ se destina para autoconsumo, el 16,5\% para incubación y el $23,5 \%$ restante para la venta. Los huevos incubados alcanzan el $24 \%$ de eclosión, y provienen de aves alimentadas básicamente con maíz, alimento comercial y acceso a pastoreo. El índice de mortalidad es de $26 \%$ y las principales enfermedades que la ocasionan son las afecciones respiratorias, las diarreas y la viruela aviar. El tratamiento cuando estas se presentan se hace, sin prescripción médico veterinaria, con oxitetraciclina, ambramicina, limón y cañaguate (Tecoma spectabilis).

Las familias de este grupo consumen aproximadamente 167 huevos per cápita año. El costo de producción de un huevo es de USD\$ 0,0092 por concepto de alimentación, y el costo aproximado de la mano de obra por gallina alcanza a los USD\$ 0,5385 al mes.

Las mujeres responsables del cuidado de las aves son amas de casa con una edad promedio de 47 años, las que manejan sus gallinas en corrales improvisados, sin especificaciones técnicas, construidos en materiales de la región y mallas metálicas. Su pretensión como alternativa económica es mantener una parvada de 40 gallinas.

\section{Grupo 4. Sistema finca de pequeñas extensiones dedicado a la producción de caña con un capital pecuario representado en un $18,6 \%$ en aves}

Este grupo se compone del $17 \%$ de las fincas encuestadas, las propiedades tienen un área promedio de 2,2 ha. Los ingresos brutos generados por la actividad pecuaria ascienden a USD\$ 242,1 anuales aproximadamente, correspondientes al 20,23\% de los ingresos brutos del sector agropecuario, del cual la especie avícola con USD\$44,30 representa el 18,3\% y los porcinos aportan el $11,6 \%$.

En este grupo las gallinas también presentan características de semipesadas, se manejan en parvadas de 12 aves, se alimentan básicamente de maíz, y algo de alimento balanceado comercial. Además se les suministra caña de azúcar (Saccharum officinarum) picada como fuente de energía, bore (Alocasia macrorrhiza) o quiebra barrigo (Trichanthera gigantea) como fuente proteica y se hace la práctica habitual de pastoreo. Las aves, tanto hembras como machos, tienen en promedio $1,8 \mathrm{~kg}$ de peso vivo, son animales jóvenes que no han completado su 
crecimiento; el 98\% de estas aves provienen de la incubación natural en la misma finca, tienen un índice de eclosión del 84\% y la madurez sexual la adquieren a los siete meses de edad. El índice de mortalidad es del $26 \%$ al año, siendo responsables afecciones como "el moquillo" o coriza, la viruela aviar y el Newcastle, se usan antibióticos como la oxitetraciclina y penicilina para tratar las enfermedades; el limón también se utiliza con fines terapéuticos, no se hace ningún tipo de tratamiento preventivo, la vacunación no es una práctica común.

El porcentaje de producción de las aves es de aproximadamente el 50\% que representa 126 huevos al mes, con peso promedio de $61 \mathrm{~g}$, el $52 \%$ de la producción de huevos se destina para autoconsumo, el $17 \%$ para la incubación y el 31\% para la venta. El costo de producción de un huevo es de USD\$ 0,133 representado principalmente en la alimentación, y el costo de la mano de obra es de USD $\$ 0,533$ por gallina que corresponde al tiempo que dedican al cuidado de esta especie al mes.

Las señoras encargadas de las gallinas tienen una edad promedio de 52 años, manejan sus gallinas en un sistema extensivo, utilizan como gallineros jaulas o árboles al aire libre, tienen los nidos construidos dentro de las viviendas, el agua y la comida les ofrecen en recipientes improvisados. Las familias de este grupo no compran huevos, tienen un consumo de 131 huevos per cápita año, ven en la explotación avícola una fuente de ingresos, la prioridad es tener una parvada de 28 gallinas criollas.

\section{Grupo 5. Sistema finca de medianas extensiones dedicado a la producción de café y ganado bovino, la explotación avícola representa el $6,3 \%$ del capital pecuario}

Este grupo reúne el $8,5 \%$ de las fincas que tienen un área promedio de 9 ha. El capital pecuario existente en la finca asciende a USD\$ 316,2 que representa el 52,16\% del total de la producción agropecuaria, siendo los bovinos los que representan el $87,8 \%$, las gallinas el $6,3 \%$ y el $5,8 \%$ los cerdos.

En este grupo prevalecen parvadas pequeñas de 4 a 5 gallinas, con características fenotípicas de semipesadas, peso a la edad adulta de 2,0 kg para las hembras y 2,7 kg para los machos. No se tiene en cuenta ningún criterio de selección, aunque el $85 \%$ de estas aves provienen de la incubación en la misma finca, en la que se logra una eclosión del 77,5\%. La producción de huevo es del $42,5 \%$ con peso medio unitario de $59 \mathrm{~g}$ y un costo de producción de USD\$ 0,16. Los costos por manejo de estas aves llegan a USD\$ 1,18 por gallina al mes. No se contempla un plan sanitario y no se aplican medidas preventivas. Las enfermedades como la viruela aviar y la coriza, que son las principales entidades patológicas responsables por la mortalidad del 23\%, son tratadas con algunos antibióticos y limón, sin prescripción médico veterinaria.

Las mujeres de este grupo tienen en promedio 36 años de edad y su objetivo es llegar a 70 gallinas, sean criollas o comerciales. 
Compran mensualmente de 30 a 35 huevos y venden una gallina en el mismo periodo. El consumo de huevo está en 105 huevos per cápita año.

Grupo 6. Sistema finca de medianas extensiones dedicado a la producción ganadera, con el $2,8 \%$ del capital pecuario representado en aves

Este grupo reúne el $10,6 \%$ de las fincas encuestadas. Los predios tienen un área promedio de 8 ha. El capital pecuario se avaluó en USD\$945,3 el renglón más representativo es el ganado bovino que aporta el 95,9\%, seguido de las gallinas con el $2,93 \%$ y los cerdos con el $1,17 \%$. En este grupo aunque la gallina no es un rubro importante, la población existente satisface la demanda de huevo del núcleo familiar. Son comunes las parvadas de siete a ocho aves, obtenidas por incubación en la misma finca. La producción de huevos alcanza el 59,1\%, con peso medio de $57 \mathrm{~g}$ por huevo, y un costo unitario de producción de USD\$ 0,0092. El costo mensual por ave es de USD\$ 0,586. En este grupo no se tiene establecido para las aves planes de vacunación ni de vermifugación, razón por la cual enfermedades como la viruela aviar, Newcastle y el parasitismo causan el $19,4 \%$ de mortalidad. Los tratamientos curativos, ocasionalmente, se hacen con oxitetraciclina, penicilina y limón.

La edad promedio de las mujeres encargadas de las gallinas es de 37 años, tienen como meta mantener una parvada de 21 gallinas, como alternativa económica. Estas familias consumen una gallina al mes, comprada en la misma región y 196 huevos per cápita año. 
Tabla 1. Materias alimenticias suministradas a las aves en la zona de influencia de la Selva de Florencia, departamento de Caldas, Colombia

\begin{tabular}{|c|c|c|c|c|}
\hline Alimento & Presentación & $\begin{array}{l}\text { Forma de } \\
\text { suministro }\end{array}$ & $\begin{array}{l}\text { Tipo de ave que } \\
\text { lo consume }\end{array}$ & Disponibilidad \\
\hline $\begin{array}{l}\text { Desperdicios } \\
\text { de cocina }\end{array}$ & Revuelto & Cocido & $\begin{array}{c}\text { Suministrado a } \\
\text { aves detodas las } \\
\text { edades }\end{array}$ & Todo el año \\
\hline \multirow[t]{2}{*}{ "Patâno" } & $\begin{array}{l}\text { "Pülpa del"' } \\
\text { fruto }\end{array}$ & "Crudo maduro" & $\begin{array}{l}\text { Gallinas aduitasy } \\
\text { pollos en levante }\end{array}$ & \multirow[t]{2}{*}{ Todo el año } \\
\hline & & Verde cocidó & Gallinas adültas & \\
\hline \multirow[t]{2}{*}{ Maiz } & Grano & Entero & $\begin{array}{c}\text { Gallinas y pollos } \\
\text { en levante }\end{array}$ & \multirow[t]{2}{*}{ Todo el año } \\
\hline & & Molido. & $\begin{array}{l}\text { Para pollitos y } \\
\text { pollos en levante }\end{array}$ & \\
\hline $\begin{array}{l}\text { Balanceado } \\
\text { comercial }\end{array}$ & "crombo & $\begin{array}{l}\text { Harina o } \\
\text { crombelizado }\end{array}$ & Gallinas & $\begin{array}{l}\text { "Disponible pero } \\
\text { la utilización no } \\
\text { es frecuente } \\
\text { (época) }\end{array}$ \\
\hline "Salvadó & Harina & $\begin{array}{l}\text { Humedo y } \\
\text { revuelto con } \\
\text { otros productos }\end{array}$ & Gallinas & $\begin{array}{l}\text { No muy } \\
\text { frecuente }\end{array}$ \\
\hline cachaza & Líquida & Liquida & "Gallinas" & $\begin{array}{l}\text { Disponible en } \\
\text { época de } \\
\text { molienda }\end{array}$ \\
\hline "Caña" & Pülpa & "Picada" & "Gallinas" & Todo el año \\
\hline Bore & Hoja" & "Picada" & "'Gallinas adultas" & Todo el ano" \\
\hline Nacedero & Hoja & "Micada" & Gallinas adultas & Todoelano \\
\hline
\end{tabular}

Fuente: Esta investigsción. 
Tabla 2. Recursos de origen vegetal recolectados por las gallinas en la zona de influencia de la Selva de Florencia, departamento de Caldas, Colombia

\begin{tabular}{|c|c|c|c|c|}
\hline Nombre vulgar & Nombre cientifico & Familia & $\begin{array}{c}\text { Parte } \\
\text { ofrecida }\end{array}$ & $\begin{array}{l}\text { Tipo de } \\
\text { planta }\end{array}$ \\
\hline Bija & Bóa Orellana Linn & Bixaceae & Semilla & Arbusto \\
\hline "Bledo. & Amaranthus cf. cruentus $L$. & Amaranthaceae & "E." & Hierba" \\
\hline "Botonallo" & Acmeila brachygiossa Cass & Asteraceae & "E.". & Hierba \\
\hline "Botoncillon & Aspina sp. & Asteraceae & Flor & Hierba \\
\hline "Churco & "Begonia semiovata Liebm" & "Begoniaceae & "E.". & Hierba" \\
\hline "Descansel" & Afernantheralanceolata(Benth)' & Amaranthaceae & Hoja & Hierba" \\
\hline Dona juana & Adenostemma lavenia (C.) Ktze & Asteraceae & Hoja & Arbusto \\
\hline "Escobabosa" & "Sida rhombiroila L.. & Malvaceae" & H.F.". & Hierba" \\
\hline Hojablanca & Calathea rutea (Aubl.) Schultes & Maranthaceae & Hoja & Hierba" \\
\hline Limoncillo" & $\begin{array}{c}\text { Cymbopogon citratus (D.C) } \\
\text { Stapf }\end{array}$ & Gramineae & "Hoja & "Hierba" \\
\hline "Paico" & Chenopodium ambrosioides L.' & Chenopodiaceae & "E.". & "Hierba" \\
\hline "Platano" & "Musa sp." & Musaceae & Hoja & Hierba" \\
\hline "Poleo & Satureia brownei (Sw.)"Bric & Lamiaceae & P.E. & Hierba" \\
\hline Quinceanera & Cuphea striguiosa H.В.К. & Lytraceae & "Flor & "Hierba" \\
\hline $\begin{array}{l}\text { Santa maria de } \\
\text { anis }\end{array}$ & Piper auritum H.B.K. & "Piperaceae & Hoja" & Arbusto \\
\hline Uchuva" & Physalis peruviana L." & Solanaceae & "E.". & Hierba" \\
\hline Venturosa & Lantana camara L. & Verbenaceae & "F."Н." & Arbusto \\
\hline Yerba buena & Wentha cf. viridis $\mathrm{L}$. & Lamiaceae & "E.". & "Hierba" \\
\hline Yerba demora & "Solanumnigrum Martens \& Gol.' & Solanaceae & H.F. & Hierba" \\
\hline Yerbalachiva & Ageratum conyzoides $L$. & Asteraceae & "F.".". & Hierba" \\
\hline
\end{tabular}

P.E.: Plants Enters; H.F.: Hojs y Fruto; F.H.: Flory Hojs.

Fuente: Esta investigscion.

\section{DISCUSIÓN}

Las gallinas hacen parte de las especies de huerta, la cría de las aves es realizada por mujeres en un sistema de crianza extensivo en el que durante la noche son confinadas en gallineros para su protección contra depredadores. El 100\% de la población es para autoconsumo, fenómeno que posiciona a este tipo de explotación en un lugar importante dentro de las familias rurales desde el punto de vista socioeconómico, para ayudar a reducir el gasto de la familia en la compra de alimentos y favorecer el arraigo del aspecto cultural.

Las parvadas más numerosas se encuentran en los grupos de fincas 3 y 4 , pero en general, están integradas en promedio por 7 u 8 gallinas, situación que se repite en zonas rurales de México y del Valle del Cauca en Colombia (Valencia y Betancourth, 1991; Palma y Vargas, 2000), esto demuestra que la explotación de la gallina criolla es común en las familias campesinas de los países latinoamericanos, pero que como resultado de la poca importancia que se le ha dado, no suple las necesidades de los 
núcleos familiares conformados por cinco o más personas. En cambio, en Puerto Rico, familias indígenas que mantienen parvadas de 10 a 15 gallinas, generan ingresos económicos y están acordes con el consumo promedio per cápita de huevos del país (Lok y Méndez, 1998). Con respecto a la interacción entre el área del predio y el capital pecuario, las gallinas hacen mayor aporte al capital y se tornan más importantes como principal fuente de alimento a medida que disminuye el área de la finca y las familias poseen menos extensión de tierras.

El sistema de producción usado por la totalidad de las familias, es la cría extensiva con el propósito de abaratar costos. Las dietas de las aves están compuestas de una amplia variedad de productos, como los desperdicios de cocina, maíz, plátano, caña de azúcar picada, etc., como fuentes de energía complementadas con alimentos que las aves recolectan en las áreas de pastoreo, como arañas, lombrices, insectos y ranas, que aportan la proteína; este mismo sistema es usado por el $65 \%$ de los agricultores en el Valle del Cauca y en el $100 \%$ de los sistemas aplicados por las comunidades del Pacífico colombiano (Valencia y Betancourth, 1991; Álvarez, 1998). Estos sistemas productivos se han generalizado principalmente porque las aves se han adaptado a condiciones adversas de ladera, humedad y sombra en zonas del trópico, lo que les ha permitido desarrollar la capacidad de ser autosuficientes en la consecución del agua y alimento (Leal y Lok, 1998).

En cuanto a la cantidad del alimento, los agricultores ofrecen diariamente entre 20 y $30 \mathrm{~g}$ de maíz a cada ave, cantidad que representa el $80 \%$ de la ración, la cual se distribuye en dos comidas. Este tipo de dieta genera competición directa entre aves y cerdos, causada por la dependencia del mismo alimento de las dos especies, lo que hace que el impulso a una de las especies incurra en disminución de la otra, que según Figueroa y Rodríguez (1994) y Vargas (1996) explican el porqué el cerdo sea la especie que tiene la menor participación en el capital pecuario, mostrándose menos importante, como observado en la zona de estudio.

El manejo sanitario de las aves bajo las condiciones de las familias campesinas es muy precario, hacen tratamiento de enfermedades con remedios caseros, no se aplican los programas de vacunación por desconocimiento y deficiente disponibilidad de productos; las afecciones respiratorias, la diarrea, el Newcastle y los parásitos externos e internos son las principales causas de mortalidad en los grupos 1, 3 y 4 , con un promedio del $23 \%$. Los resultados observados se encajan dentro de los índices de mortalidad para estos tipos de sistemas de producción, caracterizados por ser muy variables y estar estrechamente relacionados con la edad de los animales en ocasiones llegando al 50 o 70\% hasta las 2 semanas de edad y al 30 o 40\% en aves adultas (Palma y Vargas, 2000; Xavier et al., 2011; Jacob et al., 2014).

Entre los parámetros productivos, la madurez sexual o edad al primer huevo, se logra entre los 180 y 210 días. El peso 
promedio de los huevos es de $58 \mathrm{~g}$, con un peso corporal de las hembras y machos en promedio de 1,9 y 2,1 kg, respectivamente. Estos parámetros se encuentran dentro del intervalo encontrado en México entre 120 y 365 días, peso promedio del huevo de $50 \mathrm{~g}$ y peso corporal de los animales de 1,8 a $1,9 \mathrm{~kg}$ a la edad de 15 meses (Palma y Vargas, 2000). El promedio de producción fue del $46,3 \%$ siendo el grupo 6 el de mayor índice con $59,1 \%$ y el grupo 2 el de menor producción con $28 \%$. El período de pausa ovárica $29,7 \%$ y $19,6 \%$ de días de descanso, respectivamente (Juárez-Caratachea y Ortiz, 2001). Los mayores índices de eclosión se presentan en los grupos 4, 5 y 6 con $84 \%, 77,5 \%$ y $73,8 \%$, respectivamente, y en el grupo 3 el menor índice con el 24\%, para un promedio general de $61,5 \%$, que coinciden con los resultados de Juárez-Caratachea y Ortiz (2001), quienes al incubar huevos de gallinas criollas recolectados en zonas rurales de México, con similar estructura genética obtuvieron $58,5 \%$ de eclosión.

Con respecto a las personas encargadas a la actividad avícola, se encontró que en el $100 \%$ de las fincas, las aves están al cuidado de la señora de la casa, cuyas habilidades han sido heredadas de sus ancestros y mejoradas con sus experiencias, sin haber recibido nunca capacitación técnica de entes estatales o instituciones privadas sobre la crianza de aves. Wieman y Leal (1998) sostienen que a la mujer campesina se le debe considerar un componente del ecosistema Andino, porque es la responsable no solo de resaltar el valor de los recursos genéticos en los Andes, sino también en la función que cumple en la conservación y utilización de la biodiversidad.

Estos resultados parecen evidenciar la poca importancia dada a las gallinas criollas, a las que se desprecia y se permite su desaparición por incompatibilidad con la moda o por la intensa propaganda a favor de tipos exóticos mejorados, sin previo estudio de las ventajas y riesgos de su producción.

Con respecto a la producción de huevos obtenida por los agricultores, el $65 \%$ de esta se destina para autoconsumo, superior en el $18 \%$ al encontrado por Valencia y Betancourth (1991) en el Valle del Cauca. En cuanto al consumo de huevos per cápita anual, los grupos 6 con 195 huevos y 3 con 167 son los de mayor índice, mientras que los grupos 2 y 5 con 101 y 105 unidades respectivamente, registran los menores consumos. El promedio en general para la zona es de 138 unidades per cápita año, inferior al promedio nacional en 90 unidades, para el año 2012 (Fenavi, 2014). Según Laredo y Cuesta (1990), el valor biológico del huevo es de 93,7\%, superior al de la leche, del pescado, carne, fríjol, soya y leguminosas seca, circunstancia que pone a los agricultores en una posición privilegiada, por disponer de este producto en sus fincas. 


\section{CONCLUSIONES}

En la zona de estudio las especies animales explotadas por el agricultor tienen relación entre sí, de tal manera que el impulso que se le da a una de ellas deprime el desarrollo de la otra, como es el caso de las aves domésticas y los cerdos los cuales compiten por el mismo alimento, situación que explica el porqué en las fincas estudiadas, los cerdos nunca fueron más importantes que las gallinas.

La cría de gallinas es una actividad arraigada dentro de la familia campesina, debido a la facilidad con que se desarrollan estos animales, al corto periodo de crecimiento en comparación con los cerdos, bovinos y ovinos, así como, por la baja inversión de capital inicial que se requiere para su explotación, además del alto grado de adaptación que han alcanzado estos animales a las condiciones climáticas adversas.

La avicultura de traspatio es una actividad importante en las comunidades rurales, que se complementa con las demás prácticas productivas realizadas en el campo, aporta nutrientes a la dieta cumpliendo la función de transformar productos de origen vegetal y residuos en proteína de origen animal; y está en estrecha relación con el área de la finca, teniendo mayor importancia a medida que el área de la finca disminuye.

El manejo sanitario de las gallinas en esta zona rural es precario, los campesinos hacen tratamientos de enfermedades con medicamentos caseros, no vacunan ni desparasitan, quedando a merced de las afecciones respiratorias, la diarrea y el Newcastle, principales causas de los altos índices de mortalidad. Los productores no reciben asistencia médico veterinaria y desconocen la norma sanitaria nacional y los puntos críticos para mantener la bioseguridad de la finca.

Con respecto a los índices de producción y eclosión, son buenos, si se tienen en cuenta las deficiencias alimenticias a las que son sometidas las aves, en comparación con los parámetros que se manejan en la avicultura industrial. En cambio, el pobre índice de crecimiento y las altas tasas de mortalidad son el reflejo del bajo nivel tecnológico en que se encuentra esta actividad productiva, por lo que el futuro del agroecosistema de traspatio como alternativa para generar alimento es incierto.

\section{AGRADECIMIENTOS}

Los autores expresan sus agradecimientos a la Vicerrectoría de Investigaciones y Postgrados de la Universidad de Caldas por la financiación del proyecto. 


\section{REFERENCIAS}

- Álvarez, M. (1998). Sistema tradicional de alimentación de gallinas y patos en una población del Pacífico colombiano. Universidad de Antioquia, Medellín.

- Ballesteros, H., López, M.P., Arroyave, F. et al. (2006). Plan de manejo 2006-2010, Parque Nacional Natural Selva de Florencia. Manizales: Parques Nacionales Naturales de Colombia, Dirección Territorial Noroccidente.

- Corpocaldas y Universidad de Caldas. (2001). Informe final sobre biodiversidad de la Selva de Florencia. Manizales: Universidad de Caldas.

- Farrel, D. (2014). Función de las aves de corral en la nutrición humana. En: Revisión del Desarrollo Avícola en los Países en Desarrollo (pp. 86-89). Organización de las Naciones Unidas para la Alimentación y la Agricultura -FAO-. Recuperado el 19 de mayo de 2014 de http://www.fao.org/docrep/016/al709s/al709s00.pdf

- FENAVI -Federación Nacional de Avicultores-. (2014). Consumo Per Cápita. Recuperado el 24 de febrero de 2014 de http://fenavi.org/index.php?option=com_content\&view=articl e\&id $=2160$ \& $\mid$ temid $=556$

- Figueroa, V. y Rodríguez, J. (1994). Un alimento seco para aves basado en mieles de caña de azúcar. Livestock Research for Rural Development, 6(1): 74-80.

- Jacob, J.P., Wilson, H.R., Miles, R.D., Butcher, R.D., Mather, F.B. (2014). Factors Affecting Egg Production in Backyard Chicken Flocks. Publication \#PS-35, 1-8. University of Florida. Recuperado el 19 de mayo de 2014 de http://edis.ifas.ufl.edu/ps029

- Juárez-Caratachea, A. y Ortiz, M. (2001). Estudio de la incubabilidad y crianza en aves criollas de traspatio. Veterinaria México, 32(1): 27-32.

- Laredo, M. y Cuesta, A. (1990). Tabla de contenido nutricional en productos y subproductos agroindustriales. Instituto Colombiano Agropecuario -ICA-. Bogotá.

- Leal, D. y Lok, R. (1998). Aspectos del manejo integral de animales menores en huertos caseros indígenas y no-indígenas de Costa Rica. Programa Nacional para el Hábitat Indígena. Proyecto CATIE/GTZ y PRONAHVI, Turrialba, Costa Rica. Recuperado http://orton.catie.ac.cr/repdoc/A7167E/A7167E.PDF

- Lok, R. y Méndez, V. (1998). El uso del ordenamiento local del espacio para una clasificación de huertos en Nicaragua. En: Huertos caseros tradicionales de América Central: características, beneficios e importancia, desde un enfoque multidisciplinario. Turrialba, Costa Rica: CATIE/AGUILA/IDCR/ETC.

- Palma, R. y Vargas, M. (2000). Resumen. Tentzo. Colegio de posgraduados campus Puebla, México. Recuperado de http.//www.usuariosarnet.com.ar/mmorra/CPNP/Principal.Html

- Pym, R. (2013). Genética y cría de aves de corral en los países en desarrollo. Contribución de los genotipos autóctonos a la producción y consumo de carne de aves de corral y huevos. En: Revisión del desarrollo avícola en los países en desarrollo (pp. 90-91). Organización de las Naciones Unidas para la Alimentación y la Agricultura -FAO-. 
- Rivera, B. y Rivera, A. (1995). Florencia: una historia para contar. Florencia, Caldas: Fundación Eduquemos.

- Rodríguez, P. y Carvajal, G. (1998). Caracterización de sistemas de producción. Programa regional de investigación en sistemas de producción. CORPOICA.

- SAS -Statistical Analysis System-. (2008). User's Guide: Statistics. Inst. Inc., Cary, NC, USA.

- Valencia, N. y Betancourth, L. (1991). Origen desarrollo y descripción de gallina criolla existentes en varios municipios del Valle del Cauca. Tesis de grado. Universidad Nacional, Sede Palmira.

- Vargas, J. (1996). Estudio de las características nutricionales y el potencial productivo de recursos campesinos para la alimentación animal en el Valle del Cauca. Centro para la Investigación en Sistemas Sostenibles de Producción Agropecuaria -Cipav-. Cali, Colombia.

- Wieman, A. y Leal, D. (1998). La cría de animales menores en los huertos caseros. En Rossana Lok (Ed.), Huertos caseros tradicionales de América Central: características, beneficios e importancia, desde un enfoque multidisciplinario (pp. 85-115). Turrialba, Costa Rica.

- Xavier, J., Pascal, D., Crespo, E, Schell, H.L., Trinidad, J.A. y Bueno, D.J. (2011). Seroprevalence of Salmonella and Mycoplasma infection in backyard chickens in th estate of Entre Ríos in Argentina. Poult Sci., 90(4): 746-51.

1. M.Sc. Esp. en Sistemas de Producción Agropecuaria. Agricultura para el Departamento de Nariño -AGDENAR-. Pasto, Colombia. jltovarparedes@gmail.com

2. Ph.D. M.Sc. Profesor Departamento de Salud Animal, Grupo de investigación en Nutrición, Metabolismo y Seguridad Alimentaria. Universidad de Caldas, Manizales, Colombia. wnarvaez@ucaldas.edu.co

3. Estudiante Programa de Medicina Veterinaria y Zootecnia, Universidad de Caldas, Manizales.

Para citar este artículo: Tovar-Paredes, J.L., Narváez-Solarte W., Agudelo-Giraldo I. (2014). Tipificación de la gallina criolla en los agroecosistemas campesinos de producción en la zona de influencia de la selva de Florencia (Caldas). Revista Luna Azul, 41, 57-72. Recuperado de http://lunazul.ucaldas.edu.co/index.php?option=content\&tas k=view\&id=1055 